TITLE:

\title{
Supersymmetric quantum-Hall effect on a fuzzy supersphere
}

$\operatorname{AUTHOR}(S)$ :

Hasebe, $\mathrm{K}$

CITATION:

Hasebe, K. Supersymmetric quantum-Hall effect on a fuzzy

supersphere. Physical Review Letters 2005, 94(20): 206802.

\section{ISSUE DATE:}

2005-05-27

URL:

http://hdl.handle.net/2433/50483

RIGHT:

Copyright 2005 American Physical Society 


\title{
Supersymmetric Quantum-Hall Effect on a Fuzzy Supersphere
}

\author{
Kazuki Hasebe \\ Yukawa Institute for Theoretical Physics, Kyoto University, Kyoto 606-8502, Japan
}

(Received 30 November 2004; published 23 May 2005)

\begin{abstract}
Supersymmetric quantum-Hall liquids are constructed on a supersphere in a supermonopole background. We derive a supersymmetric generalization of the Laughlin wave function, which is a ground state of a hard-core $\operatorname{OSp}(1 \mid 2)$ invariant Hamiltonian. We also present excited topological objects, which are fractionally charged deficits made by super Hall currents. Several relations between quantum-Hall systems and their supersymmetric extensions are discussed.
\end{abstract}

DOI: 10.1103/PhysRevLett.94.206802

Quantum-Hall systems contain noncommutative structures, such as Matrix theories and D-brane physics [1]. They are perhaps the simplest known physical setup of noncommutative geometry and exhibit many of its exotic properties [2]. Therefore, quantum-Hall systems, which can be readily investigated in the laboratory, represent a practical alternative to the physics which string theory attempts to describe, which is still far beyond our realm of experimental capability. This is one reason that quantum-Hall systems are very fascinating. It is expected that ideas developed by investigating quantum-Hall systems will help in furthering our understanding of the high energy physics [3].

Originally, quantum-Hall phenomena were realized in the two-dimensional flat space under strong magnetic field. Laughlin derived a wave function that well describes quantum incompressible liquids [4]. His wave function is rotationally symmetric but not translationally symmetric on the plane. Hence, it does not possess all the symmetries in a plane and is not suited for computer simulations. Haldane overcame this problem by constructing quantum-Hall systems on two-spheres in a Dirac magnetic monopole background [5]. He constructed a Laughlin-like wave function, which we call the Laughlin-Haldane wave function, on the sphere that possesses all the rotational symmetries of the two-sphere. The sphere used in by Haldane's analysis is simply a fuzzy two-sphere. Recently, Zhang and Hu have succeeded in constructing four-dimensional quantum-Hall systems in a $S U(2)$ Yang monopole background [6]. The systems they consider are quantum-Hall liquids on fuzzy four-spheres and, intriguingly, possess branelike excitations. Because Matrix theories can be used to describe higher-dimensional spaces and possess extended objects, their quantum-Hall systems are the first discovered "physical" systems that exhibit behavior similar to that described by Matrix models. Their theory has attracted much attention and has been developed by many authors [7]. In particular, on the basis of fuzzy complex projective manifolds, Karabali and Nair have generalized them into even higher-dimensional quantum-Hall systems [8]. Hasebe and Kimura, based on higher-dimensional fuzzy spheres, have found another way to generalize them for an arbitrary even
PACS numbers: 73.43.-f, 02.40.Gh, 11.10.Nx, 11.30.Pb

number of dimensions in colored monopole backgrounds [9]. In fact, such developments in the study of quantumHall systems have provided information that may be important in obtaining an understanding of D-brane physics. In particular, it has been reported that, with use of the Dirac-Born-Infeld action, the higher-dimensional fuzzy spheres in Matrix models can be identified with dielectric D-branes in colored monopole backgrounds [10].

Recently, it was found that nonanticommutative (NAC) field theory is naturally realized on D-branes in $R$ - $R$ field or graviphoton backgrounds [11]. Also, it has been shown that, in supermatrix models, fuzzy superspheres arise as classical solutions, and their fluctuations yield NAC field theories [12]. Some interesting relations between lowest Landau level (LLL) physics and NAC geometry have also been reported [13]. With these recent developments, it would be worthwhile to extend the theory of quantumHall systems to a supersymmetric framework. Indeed, the supersymmetric quantum-Hall systems might be the simplest physical setup of NAC geometry. Further, encouraged by previous success in the investigation of higherdimensional quantum-Hall systems, we may hope that such systems not only possess exotic properties in the NAC world but also reveal yet unknown aspects of supermatrix models.

A supersphere is a geometrical object taking the form of a coset manifold given by $S^{2 \mid 2}=O S p(1 \mid 2) / U(1)$. By construction, a supersphere manifestly possesses the exact $\mathcal{N}=1$ supersymmetry, which is generated by the $\operatorname{OSp}(1 \mid 2)$ super Lie group. The fact that the supersymmetry remains exact is an advantage of using the coset manifolds of super Lie groups. The number of degrees of freedom of the supersphere is given by $\operatorname{dim} S^{2 / 2}=$ $\operatorname{dim} \operatorname{OSp}(1 \mid 2)-\operatorname{dim} U(1)=5-1=4$. Two of these degrees of freedom correspond to the Grassmann even coordinates, and the other two correspond to the Grassmann odd coordinates on the supersphere. The supersphere is embedded in a flat superspace whose coordinates are $x_{a}(a=1,2,3)$, which are Grassmann even, and $\theta_{\alpha}(\alpha=$ $1,2)$, which are Grassmann odd. The radius of the supersphere is given by $R=\sqrt{x_{a}^{2}+C_{\alpha \beta} \theta_{\alpha} \theta_{\beta}}$, where $C_{\alpha \beta}$ is an antisymmetric tensor with $C_{12}=1$. At the center of the 
supersphere, we place a supermonopole whose magnetic charge is $I / 2$ when $I$ is an integer. The magnetic field of the supermonopole is given by $B=2 \pi I / 4 \pi R^{2}=I / 2 R^{2}$, and the magnetic length is defined as $\ell_{B} \equiv 1 / \sqrt{B}=R \sqrt{2 / I}$. The thermodynamic limit corresponds to $R, I \rightarrow \infty$, with $\ell_{B}$ fixed to a finite value. For simplicity, in the following we set $R=1$, which makes all quantities dimensionless.

We now briefly discuss the one-particle state on the supersphere in the supermonopole background. A detailed analysis is found in Ref. [14]. The one-particle Hamiltonian is given by

$$
H=\frac{1}{2 M}\left(\Lambda_{a}^{2}+C_{\alpha \beta} \Lambda_{\alpha} \Lambda_{\beta}\right)
$$

where $\Lambda_{a}$ and $\Lambda_{\alpha}$ are the $\operatorname{OSp}(1 \mid 2)$ covariant particle angular momenta, $\Lambda_{a}=-i \epsilon_{a b c} x_{b} D_{b}+\frac{1}{2} \theta_{\alpha}\left(\sigma_{a}\right)_{\alpha \beta} D_{\beta}$ and $\Lambda_{\alpha}=\frac{1}{2}\left(C \sigma_{a}\right)_{\alpha \beta} x_{a} D_{\beta}-\frac{1}{2} \theta_{\beta}\left(\sigma_{a}\right)_{\beta \alpha} D_{a}$. The covariant derivatives are given by $D_{a}=\partial_{a}+i A_{a}$ and $D_{\alpha}=\partial_{\alpha}+$ $i A_{\alpha}$, where $A_{a}=-\frac{I}{2} \epsilon_{a b 3} \frac{x_{b}}{1+x_{3}}\left(1+\frac{2+x_{3}}{2\left(1+x_{3}\right)} \theta C \theta\right)$ and $A_{\alpha}=$ $-\frac{I}{2} i\left(\sigma_{a} C\right)_{\alpha \beta} x_{a} \theta_{\beta}$. The supermonopole field strengths are given by $B_{a}=-\frac{I}{2} x_{a}$ and $B_{\alpha}=-\frac{I}{2} \theta_{\alpha}$. The commutation relations for the covariant angular momenta of the particle are obtained as $\left[\Lambda_{a}, \Lambda_{b}\right]=i \epsilon_{a b c}\left(\Lambda_{c}-B_{c}\right),\left[\Lambda_{a}, \Lambda_{\alpha}\right]=\frac{1}{2} \times$ $\left(\sigma_{a}\right)_{\beta \alpha}\left(\Lambda_{\beta}-B_{\beta}\right)$, and $\left\{\Lambda_{\alpha}, \Lambda_{\beta}\right\}=\frac{1}{2}\left(C \sigma_{a}\right)_{\alpha \beta}\left(\Lambda_{a}-B_{a}\right)$. Thus, they do not satisfy the $\operatorname{OSp}(1 \mid 2)$ commutation relations exactly, due to the presence of the supermagnetic field. The total $O S p(1 \mid 2)$ angular momenta are constructed as $L_{a}=\Lambda_{a}+B_{a}$ and $L_{\alpha}=\Lambda_{\alpha}+B_{\alpha}$. The operators $L_{\alpha}$ play the role of the supercharge in the system. The covariance under $\operatorname{OSp}(1 \mid 2)$ transformations is expressed as $\left[L_{a}, X_{b}\right]=i \epsilon_{a b c} X_{c},\left[L_{a}, X_{\alpha}\right]=\frac{1}{2}\left(\sigma_{a}\right)_{\beta \alpha} X_{\beta}$ and $\left\{L_{\alpha}, X_{\beta}\right\}=$ $\frac{1}{2}\left(C \sigma_{a}\right)_{\alpha \beta} X_{a}$, where $X_{a}$ represents $L_{a}, \Lambda_{a}$, and $B_{a}$, and $X_{\alpha}$ represents $L_{\alpha}, \Lambda_{\alpha}$, and $B_{\alpha}$.

The $\operatorname{OSp}(1 \mid 2)$ Casimir operator for the total $\operatorname{OSp}(1 \mid 2)$ angular momenta $L_{a}$ and $L_{\alpha}$ are $L_{a}^{2}+C_{\alpha \beta} L_{\alpha} L_{\beta}=j\left(j+\frac{1}{2}\right)$, where $j=\frac{I}{2}+n$. Here, $n=0,1,2, \ldots$ indicates the Landau level. The supermonopole field is perpendicular to the surface of the supersphere, while the particle moves on the supersphere. Therefore, the particle angular momenta are orthogonal to the supermonopole field: $\Lambda_{a} B_{a}+$ $C_{\alpha \beta} \Lambda_{\alpha} B_{\beta}=B_{a} \Lambda_{a}+C_{\alpha \beta} B_{\alpha} \Lambda_{\beta}=0$. Observing these relations, we find the energy eigenvalues to be $E_{n}=\frac{1}{2 M} \times$ $\left[n\left(n+\frac{1}{2}\right)+I\left(n+\frac{1}{4}\right)\right]$. Thus, in the LLL, the energy becomes $E_{\mathrm{LLL}}=\frac{1}{4} \omega$, where $\omega=B / M$ is the cyclotron frequency.

The number of unit cells, each occupying an area $2 \pi \ell_{B}^{2}$ on the supersphere, is $N_{\Phi}=4 \pi / 2 \pi \ell_{B}^{2}=I$. It is convenient to define the filling fraction as $\nu=N / N_{\Phi}$. We now give a comment. From the $O S p(1 \mid 2)$ representation theory, the dimension of the irreducible representation $j=I / 2$ is $D=(2 j+1)+\left.(2 j)\right|_{j=I / 2}=2 I+1$. Therefore, the number of states in the LLL is twice as large as the number of magnetic cells in the large $I$ limit. This implies that in each magnetic cell, there are two degenerate states due to the supersymmetry. Hence, in this system, the value of the filling fraction $N / N_{\Phi}$ is twice as large as that in the ordinary definition $N / D$.

The supercoherent state $\psi$ is defined as the state that is aligned in the direction of the supermagnetic flux, $\left(B_{a}, B_{\alpha}\right) \propto\left(x_{a}, \theta_{\alpha}\right)$; that is, we have $l_{a} \psi \cdot x_{a}+C_{\alpha \beta} l_{\alpha} \psi$. $\theta_{\beta}=\frac{1}{2} \psi$, where $l_{a}$ and $l_{\alpha}$ constitute the fundamental representation of the $\operatorname{OSp}(1 \mid 2)$ generators. Explicitly, these are

$$
l_{a}=\frac{1}{2}\left(\begin{array}{cc}
\sigma_{a} & 0 \\
0 & 0
\end{array}\right), \quad l_{\theta_{1}}=\frac{1}{2}\left(\begin{array}{cc}
0 & \tau_{1} \\
\tau_{2}^{t} & 0
\end{array}\right), \quad l_{\theta_{2}}=\frac{1}{2}\left(\begin{array}{cc}
0 & \tau_{2} \\
-\tau_{1}^{t} & 0
\end{array}\right)
$$

where the quantities $\left\{\sigma_{a}\right\}$ are the Pauli matrices, while $\tau_{1}=(1,0)^{t}$ and $\tau_{2}=(0,1)^{t}$. Up to a $U(1)$ phase factor, the explicit form of the supercoherent state is found to be $\psi=(u, v, \eta)^{t}=\left\{\sqrt{\left(1+x_{3}\right) / 2}\left(1-\frac{1}{4\left(1+x_{3}\right)} \theta C \theta\right), \frac{x_{1}+i x_{2}}{\sqrt{2\left(1+x_{3}\right)}} \times\right.$ $\left.\left(1+\frac{1}{4\left(1+x_{3}\right)} \theta C \theta\right), \frac{1}{\sqrt{2\left(1+x_{3}\right)}}\left[\left(1+x_{3}\right) \theta_{1}+\left(x_{1}+i x_{2}\right) \theta_{2}\right]\right\}^{t}$. This is identical to the super Hopf spinor, which satisfies the relations $\psi^{\ddagger} l_{a} \psi=\frac{1}{2} x_{a}$ and $\psi^{\ddagger} l_{\alpha} \psi=\frac{1}{2} \theta_{\alpha}$, where $\ddagger$ denotes the superadjoint, defined as $\psi^{\ddagger}=\left(u^{*}, v^{*},-\eta^{*}\right)$, and $*$ denotes pseudoconjugation, which acts on a Grassmann odd number $\xi$ as $\xi^{* *}=-\xi,\left(\xi_{1} \xi_{2}\right)^{*}=\xi_{1}^{*} \xi_{2}^{*}$. The coordinates $\left\{x_{a}, \theta_{\alpha}\right\}$ are superreal, in the sense that we have $\left(x_{a}^{*}, \theta_{\alpha}^{*}\right)=\left(x_{a}, C_{\alpha \beta} \theta_{\beta}\right)$.

The supercoherent state in a supermonopole background can be obtained similarly. The supercoherent state, directed to the point $\left(\Omega_{a}, \Omega_{\alpha}\right)$, should satisfy the equation $\left[\Omega_{a}(\chi) L_{a}+C_{\alpha \beta} \Omega_{\alpha}(\chi) L_{\beta}\right] \psi_{\chi}^{(I)}(u, v, \eta)=-\frac{I}{2} \psi_{\chi}^{(I)}(u, v, \eta)$, where $\chi$ is a constant super Hopf spinor given by $\chi=$ $(a, b, \xi)^{t}$, which is mapped to the point $\left(\Omega_{a}, \Omega_{\alpha}\right)$ on the supersphere by $\Omega_{a}(\chi)=2 \chi^{\ddagger} l_{a} \chi$ and $\Omega_{\alpha}(\chi)=2 \chi^{\ddagger} l_{\alpha} \chi$. The supercoherent state is found to be $\psi_{\chi}^{(I)}(u, v, \eta)=$ $\left(\chi^{\ddagger} \psi\right)^{I}=\left(a^{*} u+b^{*} v-\eta^{*} \xi\right)^{I}$.

Supermonopole harmonics $u_{m_{1}, m_{2}}$ and $\eta_{n_{1}, n_{2}}$ are introduced on the supersphere. They form a basis for the LLL and are eigenstates of $L_{z}$ with the eigenvalues $\frac{m_{2}-m_{1}}{2}$ and $\frac{n_{2}-n_{1}}{2}$, respectively. Their explicit forms are $u_{m_{1}, m_{2}}=$ $\sqrt{I ! / m_{1} ! m_{2} !} u^{m_{1}} \boldsymbol{v}^{m_{2}} \quad$ and $\quad \eta_{n_{1}, n_{2}}=\sqrt{I ! / n_{1} ! n_{2} ! u^{n_{1}}} \boldsymbol{v}^{n_{1}} \eta$, where $m_{1}+m_{2}=I$ and $n_{1}+n_{2}=I-1$. The degeneracy of $u_{m_{1}, m_{2}}$ is $(I+1)$, while that of $\eta_{n_{1}, n_{2}}$ is $(I)$. Thus, the total degeneracy is $(2 I+1)$, which is exactly the dimension of the Hilbert space of the LLL. Thus, without including any complex variables $\left\{u^{*}, v^{*}, \eta^{*}\right\}$, the functions in the LLL are constructed from the variables $\{u, v, \eta\}$. For this reason, the $\operatorname{OSp}(1 \mid 2)$ operators are effectively represented as $L_{a}=\psi^{t} \tilde{l}_{a} \frac{\partial}{\partial \psi}$ and $L_{\alpha}=\psi^{t} \tilde{l}_{\alpha} \frac{\partial}{\partial \psi}$, where $\frac{\partial}{\partial \psi}=$ $\left(\frac{\partial}{\partial u}, \frac{\partial}{\partial v}, \frac{\partial}{\partial \eta}\right)^{t}$ and $\left\{\tilde{l}_{a}, \tilde{l}_{\alpha}\right\}$ forms a complex representation of $\operatorname{SSp}(1 \mid 2)$ with $\tilde{l}_{a}=-l_{a}^{*}$ and $\tilde{l}_{\alpha}=C_{\alpha \beta} l_{\beta}$. The complex representation in $\operatorname{OSp}(1 \mid 2)$ is related to the original by the unitary transformation, $\tilde{l}_{a}=\mathcal{R}^{t} l_{a} \mathcal{R}, \tilde{l}_{\alpha}=\mathcal{R}^{t} l_{\alpha} \mathcal{R}$, where $\mathcal{R}$ is given by 


$$
\mathcal{R}=\left(\begin{array}{ccc}
0 & 1 & 0 \\
-1 & 0 & 0 \\
0 & 0 & 1
\end{array}\right)
$$

Thus, the representation of $O S p(1 \mid 2)$ is pseudoreal. The properties of $\mathcal{R}$ are as follows: $\mathcal{R}^{t}=\mathcal{R}^{\dagger}=\mathcal{R}^{\ddagger}=\mathcal{R}^{-1}$ and $\mathcal{R}^{2}=\left(\mathcal{R}^{t}\right)^{2}=\operatorname{diag}(-1,-1,1)$. Using the matrix $\mathcal{R}$, the complex spinor is given by $\tilde{\psi}=\mathcal{R}\left(\psi^{\ddagger}\right)^{t}=$ $\left(v^{*},-u^{*}, \eta^{*}\right)^{t}$. Then, without including the complex spinor $\psi^{\ddagger}$, the $\operatorname{OSp}(1 \mid 2)$ singlet can be constructed from $\psi$ and $\psi^{\prime}$ alone: $\tilde{\psi}^{\ddagger} \psi^{\prime}=\psi^{t} \mathcal{R} \ddagger \psi^{\prime}=\left(\psi^{\prime}\right)^{t} \mathcal{R} \psi$.

We next study the two-particle state. The total angular momenta of $O S p(1 \mid 2)$ are given by $L_{a}^{\text {tot }}=L_{a}(1)+L_{a}(2)$ and $L_{\alpha}^{\mathrm{tot}}=L_{\alpha}(1)+L_{\alpha}(2)$, where $L_{a}(i)$ and $L_{\alpha}(i)$ are the $\operatorname{OSp}(1 \mid 2)$ generators of the $i$ th particle. The two-particle supercoherent state located at the point $\left(\Omega_{a}, \Omega_{\alpha}\right)$ satisfies the equation $\quad\left[\Omega_{a}(\chi) L_{a}^{\mathrm{tot}}+C_{\alpha \beta} \Omega_{\alpha}(\chi) L_{\beta}^{\mathrm{tot}}\right] \psi_{\chi}^{(I, J)}=$ $-J \psi_{\chi}^{(I, J)}$. The solution is written as

$$
\begin{aligned}
\psi_{\chi}^{(I, J)}= & \left(u_{1} v_{2}-v_{1} u_{2}-\eta_{1} \eta_{2}\right)^{I-J} \\
& \cdot \psi_{\chi}^{(J)}\left(u_{1}, v_{1}, \eta_{1}\right) \psi_{\chi}^{(J)}\left(u_{2}, v_{2}, \eta_{2}\right),
\end{aligned}
$$

where the first component on the right-hand side is an $\operatorname{OSp}(1 \mid 2)$ singlet that determines the distance between two particles. Thus, the two-particle state $\psi_{\chi}^{(I-J)}$ represents an extended object whose spin is $J$, whose center-of-mass is located at $\left(\Omega_{a}(\chi), \Omega_{\alpha}(\chi)\right)$, and whose size is proportional to $(I-J)$.

In the LLL, this system reduces to a fuzzy supersphere [14]. Hence, the two-body interaction is reduced to that on a fuzzy supersphere, which is expressed as a supersymmetric extension of Haldane's pseudopotential [5], $\Pi_{I} V\left[\Omega_{a}(1) \Omega_{a}(2)+C_{\alpha \beta} \Omega_{\alpha}(1) \Omega_{\beta}(2)\right] \Pi_{I}=$ $\sum_{J=0,1 / 2, \ldots, I} V_{J}^{(I)} P_{J}\left[L_{a}(1) L_{a}(2)+C_{\alpha \beta} L_{\alpha}(1) L_{\beta}(2)\right]$, where $P_{J}$ is the projection operator to the Hilbert space spanned by the states that form an irreducible representation of $\operatorname{OSp}(1 \mid 2) \operatorname{spin} J$. It is noted that the $O S p(1 \mid 2)$ spin $J$ takes not only integer values but also half-integer values. Physically, this implies that fermionic particles, as well as bosonic particles, appear as two-particle states in supersymmetric quantum-Hall systems.

In Haldane's work [5], the Laughlin-Haldane wave function is constructed from the $S U(2)$ singlet state, which is apparently invariant under any rotations of the two-sphere. Therefore, it is natural to use an $O S p(1 \mid 2)$ singlet wave function as the supersymmetric extension of the LaughlinHaldane wave function. Explicitly, it is given by

$$
\Psi^{(m)}=\prod_{i<j}^{N}\left[-\psi_{j}^{t} \mathcal{R} \psi_{i}\right]^{m}=\prod_{i<j}^{N}\left(u_{i} v_{j}-v_{i} u_{j}-\eta_{i} \eta_{j}\right)^{m} .
$$

When the product is expanded, it can easily be seen that the sum of the powers of $u_{i}$ and $v_{i}$ is $m(N-1)$. Also, the LLL constraint $m_{1}+m_{2}=N_{\Phi}$ for the monopole harmonics $u_{m_{1}, m_{2}}$ should be satisfied. Hence, the number of particles and the number of magnetic cells are related as $m(N-$
1) $=N_{\Phi}$. Thus, $\Psi^{(m)}$ describes a supersymmetric quantum-Hall liquid at $\nu=1 / m$ in the thermodynamic limit. The supercoherent state for $\Psi^{(m)}$, whose center is located at $\left(\Omega_{a}(\chi), \Omega_{\alpha}(\chi)\right)$, is given by $\Psi_{\chi}^{(m)}=$ $\prod_{i}^{N}\left(a^{*} u_{i}+b^{*} v_{i}-\xi^{*} \eta_{i}\right)^{I} \Psi^{(m)}$. In fact, this wave function satisfies the supercoherent equation, $\left[\Omega_{a}(\chi) L_{a}+\right.$ $\left.C_{\alpha \beta} \Omega_{\alpha}(\chi) L_{\beta}\right] \Psi_{\chi}^{(m)}=-\frac{I}{2} N \Psi_{\chi}^{(m)}$.

The supersphere contains an ordinary two-sphere as its "body." The coordinates on this body sphere are denoted $\left\{y_{a}\right\}$. These satisfy $y_{a}^{2}=1$ and are related to $\left\{x_{a}\right\}$ as $y_{a}=$ $\left(1+\frac{1}{2} \theta C \theta\right) x_{a}$. Physical interpretations are easily formulated for phenomena on the body sphere, and some calculations are reduced to the ones on the body sphere [14]. For instance, the supermagnetic flux integral on the supersphere is reduced to the ordinary magnetic flux integral for the Dirac monopole on the body sphere. Thus, the body sphere "inside" the supersphere has a Dirac monopole at its center and becomes a fuzzy sphere in the LLL. Then, it is conjectured that Haldane's original quantum-Hall systems are realized on such a fuzzy body sphere. The super Hopf spinor is rewritten $(u, v, \eta)=\left(1-\frac{1}{4} \theta C \theta\right)\left(\mu, \nu, \mu \theta_{1}+\right.$ $\left.\nu \theta_{2}\right)$, where $(\mu, \nu)=\left(\sqrt{\left(1+y_{3}\right) / 2}, \frac{y_{1}+i y_{2}}{\sqrt{2\left(1+y_{3}\right)}}\right)$ is a Hopf spinor on the body sphere [14]. By inserting this form into $\Psi^{(m)}$, the supersymmetric extension of the LaughlinHaldane wave function can be expressed in terms of the body coordinates $\left\{y_{a}\right\}$ and soul coordinates $\left\{\theta_{\alpha}\right\}$ as

$$
\begin{aligned}
\Psi^{(m)}= & \prod_{i}^{N}\left(1-\frac{m(N-1)}{4} \theta C \theta\right)_{i} \\
& \times \prod_{i<j}\left(1-m \frac{\left(\mu \theta_{1}+\nu \theta_{2}\right)_{i}\left(\mu \theta_{1}+\nu \theta_{2}\right)_{j}}{\mu_{i} \nu_{j}-\nu_{i} \mu_{j}}\right) \Phi^{(m)},
\end{aligned}
$$

where $\Phi^{(m)}$ is the ordinary Laughlin-Haldane wave function on the body sphere: $\Phi^{(m)}=\prod_{i<j}\left(\mu_{i} \nu_{j}-\nu_{i} \mu_{j}\right)^{m}$. The second term on the right-hand side yields nontrivial connections between the body sphere and the soul space. Apparently, $\Psi^{(m)}$ is a singlet with respect to $S U(2)$ transformations generated by $\left\{L_{a}\right\}$, because it is a subgroup of $\operatorname{OSp}(1 \mid 2)$. However, it is noted that, due to the second term, $\Psi^{(m)}$ does not possess the $S U(2)$ rotational symmetry of the body sphere, which is generated by $\left\{(\mu \nu)\left(-\frac{1}{2} \sigma_{a}^{*}\right) \times\right.$ $\left.\left(\frac{\partial}{\partial \mu} \frac{\partial}{\partial \nu}\right)^{t}\right\}$.

Because, in $\Psi^{(m)}$, two particles $(i, j)$ have a power at least $m$, no two particles can become so close that we have $I-m<J_{i j}$. Therefore, just as in the LaughlinHaldane case [5], $\Psi^{(m)}$ is the exact ground state of the hard-core interaction Hamiltonian, $\Pi_{I} H_{m}^{\text {int }} \Pi_{I}=$ $\sum_{i<j} \sum_{I-m<J} V_{J} P_{J}\left[L_{a}(i) L_{a}(j)+C_{\alpha \beta} L_{\alpha}(i) L_{\beta}(j)\right]$, with energy 0 , where $V_{J}>0$. This Hamiltonian is a direct generalization of Haldan's Hamiltonian, with the $O S p(1 \mid 2)$ Casimir operator replacing the $S U(2)$ one.

Because of the noncommutative algebra on the fuzzy supersphere, the super Hall currents $I_{a}=\frac{d}{d t} x_{a}$ and 
$I_{\alpha}=\frac{d}{d t} \theta_{\alpha}$ can be expressed as

$$
\begin{aligned}
I_{a} & =-i\left[x_{a}, V\right]=\alpha \epsilon_{a b c} x_{b} E_{c}-i \alpha \frac{1}{2}\left(\sigma_{a} C\right)_{\alpha \beta} \theta_{\alpha} E_{\beta}, \\
I_{\alpha} & =-i\left[\theta_{\alpha}, V\right] \\
& =-i \alpha \frac{1}{2} x_{a}\left(\sigma_{a}\right)_{\beta \alpha} E_{\beta}-i \alpha \frac{1}{2} \theta_{\beta}\left(\sigma_{a}\right)_{\beta \alpha} E_{a}
\end{aligned}
$$

where $\alpha=2 / I$, the superelectric fields $E_{a}$ and $E_{\alpha}$ are defined as $E_{a}=-\partial_{a} V$ and $E_{\alpha}=-C_{\alpha \beta} \partial_{\beta} V$, and we have used the fact that the Hamiltonian is reduced to the potential term $V$ in the LLL. It can easily be seen that the super Hall currents satisfy the equation $E_{a} I_{a}+$ $C_{\alpha \beta} E_{\alpha} I_{\beta}=0$, which expresses the orthogonality of the Hall currents and the electric fields in the supersymmetric sense. When we pierce (eliminate) the supermagnetic flux at some point on the supersphere adiabatically, superelectric fields are induced and circle the supermagnetic flux, as described by the super Faraday law. Because of the Hall orthogonality discussed above, super Hall currents flow radially from the point at which the supermagnetic flux is pierced (eliminated). As a consequence, a charged deficit (excess), which we call a quasihole (quasiparticle), is generated at this point. The corresponding operators at the point $\left(\Omega_{a}(\chi), \Omega_{\alpha}(\chi)\right)$ are given by

$$
\begin{aligned}
A^{\ddagger}(\chi) & =\prod_{i}^{N} \psi_{i}^{t} \mathcal{R}^{t} \chi(\text { quasihole }), \\
A(\chi) & =\prod_{i}^{N} \chi^{\ddagger} \mathcal{R} \frac{\partial}{\partial \psi_{i}} \text { (quasiparticle). }
\end{aligned}
$$

The quasihole operator satisfies the commutation relation $\left[\Omega_{a}(\chi) L_{a}+C_{\alpha \beta} \Omega_{\alpha}(\chi) L_{\beta}, A^{\ddagger}(\chi)\right]=\frac{N}{2} A^{\ddagger}(\chi) . \quad$ Thus, when a quasihole (quasiparticle) is created, the angular momentum in the direction of the point $\left(\Omega_{a}, \Omega_{\alpha}\right)$ increases (decreases) by $\frac{1}{2} N$. The statistics of the quasiparticles are bosonic, i.e., $\left[A^{\ddagger}(\chi), A^{\ddagger}\left(\chi^{\prime}\right)\right]=\left[A(\chi), A\left(\chi^{\prime}\right)\right]=0$ and $\left[A(\chi), A^{\ddagger}(\chi)\right]=1$. For $\nu=1 / m$, the number of particles and the number of supermagnetic fluxes are related as $N_{\Phi}=m(N-1)$, whose variation reads $\delta N=\frac{1}{m} \delta N_{\Phi}$. Because each quasiparticle corresponds to a supermagnetic flux, this relation implies that the charge of a quasiparticle is fractional, namely, $e^{*}=1 / m$, as in the original case. However, it is noted that in the present case the charge deficit is made by both the bosonic current $I_{a}$ and the fermionic current $I_{\alpha}$ on the supersphere.

On the body sphere, the supersymmetric quantum-Hall liquid reduces to an ordinary quantum-Hall liquid and the Haldane-Halperin hierarchy is realized. Because the behavior on the supersphere is mapped to that on the body sphere, it is natural to conjecture that the supersymmetric extension of the Haldane-Halperin hierarchy is realized on the supersphere, where the superquasiparticles repeatedly condense to form a hierarchy of supersymmetric quantumHall liquids.
To summarize, we have constructed a low-dimensional supersymmetric quantum-Hall system and investigated its basic properties. Similar to the original quantum-Hall systems, this system raises many interesting questions, for instance, those regarding edge excitations, anyonic objects, effective field theory, and the relation of the present system to integrable models. In a planar limit, this system reduces to a supersymmetric harmonic oscillator system [15], which is related to the Pauli Hamiltonian for a spin-1/2 particle with a gyromagnetic factor of 2 or the JaynesCummings model without interaction terms used in quantum optics. There are many real systems which show supersymmetric properties [16]. It would be worthwhile to investigate the realization of the supersymmetric holonomy, such as the Wilczek-Zee nonabelian holonomy (which generally appears in the presence of degenerate energy levels) and possible relevance to the supersymmetric quantum-Hall effects, in real systems. With regard to high energy physics, one of the most important task is to develop a higher-dimensional generalization based on supersymmetric second and third Hopf maps. Once these are constructed, we should proceed to the investigation of their relation to D-brane systems and super Twistor models.

I would like to acknowledge Yusuke Kimura for useful discussions. I am also glad to thank Taichiro Kugo, Hiroshi Kunitomo, Naoki Sasakura, and Tatsuya Tokunaga for conversations. This work was supported by JSPS.

*Electronic address: hasebe@yukawa.kyoto-u.ac.jp

[1] L. Susskind, hep-th/0101029; A. P. Polychronakos, J. High Energy Phys. 04 (2001) 011.

[2] S. M. Girvin and T. Jach, Phys. Rev. B 29, 5617 (1984); K. Moon et al., Phys. Rev. B 51, 5138 (1995); Z. F. Ezawa et al., Phys. Rev. B 67, 125314 (2003).

[3] Shou-Cheng Zhang, hep-th/0210162.

[4] R. B. Laughlin, Phys. Rev. Lett. 50, 1395 (1983).

[5] F. D. M. Haldane, Phys. Rev. Lett. 51, 605 (1983).

[6] S. C. Zhang and J. Hu, Science 294, 823 (2001).

[7] See, for example, D. Karabali et al., hep-th/0407007, and references there in.

[8] D. Karabali and V. P. Nair, Nucl. Phys. B641, 533 (2002).

[9] K. Hasebe and Y. Kimura, Phys. Lett. B 602, 255 (2004).

[10] Y. Kimura, J. High Energy Phys. 04 (2004) 058.

[11] H. Ooguri and C. Vafa, Adv. Theor. Math. Phys. 7, 53 (2003); 7, 405 (2004); J. de Boer et al., Phys. Lett. B 574, 98 (2003); N. Seiberg, J. High Energy Phys. 06 (2003) 010.

[12] S. Iso and H. Umetsu, Phys. Rev. D 69, 105003 (2004).

[13] M. Hatsuda et al., Nucl. Phys. B671, 217 (2003); E. Ivanov et al., hep-th/0311159; hep-th/0404108.

[14] K. Hasebe and Y. Kimura, Nucl. Phys. B709, 94 (2005).

[15] K. Hasebe, hep-th/0503162.

[16] G. Junker, Supersymmetric Methods in Quantum and Statistical Physics (Springer, New York, 1996), Table 9.1. 